\title{
ANÁLISE QUALI-QUANTITATIVA DA ARBORIZAÇÃO URBANA DE DOIS BAIRROS DO MUNICIPIO DO RIO DE JANEIRO POR MEIO DO GEOPROCESSAMENTO
}

\author{
QUALI-QUANTITATIVE ANALYSIS OF THE URBAN TREES OF TWO \\ NEIGHBORHOODS OF RIO DE JANEIRO CITY BY GEOPROCESSING
}

\begin{abstract}
Aloísio Werneck Pereira ${ }^{1}$, José Carlos Arthur Junior ${ }^{2}$, Bruno Araujo Furtado de Mendonça ${ }^{2}$, Celso Junius Ferreira Santos ${ }^{1}$, Rômulo Guimarães Giácomo ${ }^{1}$, Emanuel José Gomes de Araújo², Eduardo Vinícius da Silva²
\end{abstract}

\section{RESUMO}

Inventários da arborização urbana com auxílio de ferramentas de geoprocessamento são práticas fundamentais e atuais para o patrimônio arbóreo das cidades. Objetivou-se caracterizar e avaliar parâmetros quali-quantitativos do censo das árvores das ruas do bairro Grajaú e compará-los com as árvores do bairro Centro, ambos no município do Rio de Janeiro, com auxílio de ferramentas de geoprocessamento. A arborização das ruas do bairro Grajaú comparada ao Centro apresentou maior número de árvores $(2.719 \times 2.693$, respectivamente) e diversidade de espécies ( $82 \times 50$, respectivamente). As dez espécies de maior frequência representaram 73 e $83 \%$ do total de espécies, e seis delas foram similares nos dois bairros. No bairro Grajaú, as árvores mais frequentes apresentam ampla distribuição, exceto as espécies Tamarindus indica e Licania tomentosa; assim como Licania tomentosa é predominante no Centro. No bairro Grajaú 150 árvores apresentaram lesões causadas pelo cancro, e no Centro 53 árvores. Árvores com cavidade igual e maior a 30\% da circunferência foram representadas em $70,3 \%$ pela espécie Tamarindus indica no Grajaú e $80,9 \%$ por Licania tomentosa no Centro. Com a espacialização dos indivíduos foi possível observar como são distribuídas e onde estão localizados os problemas, auxiliando no planejamento e manejo da arborização urbana.

Palavras-chave: Árvores urbanas; Sistema de Informações Geográficas; Inventário; Gestão urbana

\section{ABSTRACT}

The inventories of urban afforestation with geoprocessing tools aid are fundamental and current practices for the city's tree patrimony. Our objective was to characterized and assessed the qualitative and quantitative parameters of census' trees of the Grajaú neighborhood and compare it with the trees of the central district, both in Rio de Janeiro city, with the aid of geoprocessing tools. The street trees in Grajaú neighborhood compared to the Centro neighborhood showed a large number of trees $(2,719 \times 2,693$, respectively) and species diversity $(82 \times 50$, respectively). The ten most frequent species represented 73 and $83 \%$ of the total species, and six were similar in the two neighborhoods. In the Grajaú neighborhood, the most frequent trees are widely distributed, except the species tamarindeiro (Tamarindus indica L.) and oiti (Licania tomentosa (Benth.) Fritsch); as oiti is predominant in the Centro. In the Grajaú neighborhood, 150 trees presented lesions caused by cancer, and in the Centro 53 trees. Trees with cavity equals or greater than $30 \%$ of the occurrence were represented in $70.3 \%$ by the tamarindeiro in Grajaú neighborhood species and $80.9 \%$ by oiti in the Centro. With the spatialization of individuals it was possible to observe how they are distributed and where the problems are located, helping the planning and management of urban afforestation.

Keywords: Urban trees; Geographic Information System; Inventory; Urban management.

Recebido em 04.04.2019 e aceito em 25.06.2019

1 Engenheiro Florestal, Engenheiro da Companhia Municipal de Limpeza Urbana/RJ - COMLURB/RJ, Rio de Janeiro/RJ. Email: Ioloawerneck@hotmail.com/arborio.comlurb@gmail.com/ romuloflorestal@gmail.com

2 Engenheiro Florestal, Dr., Professor do Departamento de Silvicultura, Instituto de Florestas da Universidade Federal Rural do Rio de Janeiro. Seropédica/RJ. Email: josecarlosarthurjunior@gmail.com / brunoafmendonca@gmail.com/ ejgaraujo@gmail.com/ eduvini@gmail.com 


\section{INTRODUÇÃO}

A implantação e o manejo da arborização urbana constituem-se em serviço público utilizado como estratégia de amenização de impactos ambientais adversos devido às condições de artificialidade do meio urbano, além dos aspectos ecológico, histórico, cultural, social, estético e paisagístico, que influenciam a melhoria da qualidade de vida (CEMIG, 2011). O ambiente urbano, por ser totalmente modificado pelo homem, pode não ter as condições apropriadas ao ciclo de vida das árvores. Elas devem sobreviver, crescer e ajustar-se aos espaços deixados após o estabelecimento da urbanização, não interferindo nas ruas, nas calçadas, nos estacionamentos, nas redes elétricas e de esgoto (LIMA et al., 2007). Na implantação da arborização urbana, é fundamental que haja planejamento adequado, com definição dos objetivos e das possíveis metas qualitativas e quantitativas, pois se deve ter a clareza de que a inexistência de um plano torna os processos de implantação e manejo sem efeito (FARIA et al., 2007).

A problemática para muitas cidades é que a arborização urbana não é planejada e o manejo realizado não tem sido o mais adequado (SILVA et al., 2006; REDIN et al., 2010; SILVA et al., 2016). Como reflexo, existe um aumento da queda de árvores, assim como, perda de vitalidade das mesmas, pois grande parte se encontra em idade avançada e sobrecarregada pelo estresse promovido pelo ambiente urbano (SAMPAIO et al., 2010). O manejo da arborização urbana implica no gerenciamento, com eficiência e habilidade, dos procedimentos necessários para o plantio de cada árvore, assim como do conjunto da arborização da cidade. Portanto, o planejamento da arborização ou do cultivo de árvores no meio urbano exige um processo cuidadoso que preveja os procedimentos desde sua concepção até sua implantação e manutenção (CEMIG, 2011).

A realização do inventário na arborização de ruas é o meio mais seguro de se conhecer todo o patrimônio arbóreo que uma cidade possui, fornecendo informações sobre as prioridades e as intervenções a serem realizadas (LIMA NETO et al., 2012). Um aspecto importante do inventário é quando este é realizado de forma a fornecer uma contínua atualização das informações. Como existe um grande volume de informações necessárias para o adequado manejo da arborização, é indispensável que estas estejam organizadas por meio de um sistema computadorizado.

Estes dados podem ser espacializados, analisados e utilizados no planejamento e no manejo da arborização urbana para que a necessidade de poda, os tratamentos fitossanitários ou as remoções e plantios sejam realizadas de acordo com as prioridades de intervenções. Deste modo, objetivou-se por meio do censo arbóreo, analisar quali-quantitativamente a arborização 
urbana dos bairros Grajaú e Centro do município do Rio de Janeiro, RJ, observando diversidade de espécies, riscos de queda e conflitos existentes utilizando ferramentas do geoprocessamento.

\section{MATERIAL E MÉTODOS}

O levantamento quali-quantitativo da arborização urbana foi realizado em dois bairros do município do Rio de Janeiro, Grajaú e Centro (Figura 1). No Grajaú o levantamento foi realizado entre agosto de 2016 e janeiro de 2017; e no Centro entre março de 2013 a junho de 2015, sendo estes dados obtidos do trabalho de Silva et al. (2016). O município do Rio de Janeiro apresenta clima tropical, com a estação chuvosa bem caracterizada durante o verão, e clima seco, com temperaturas amenas durante o inverno, definido como Af (clima tropical chuvoso) na classificação Köppen (ALVARES et al., 2013).

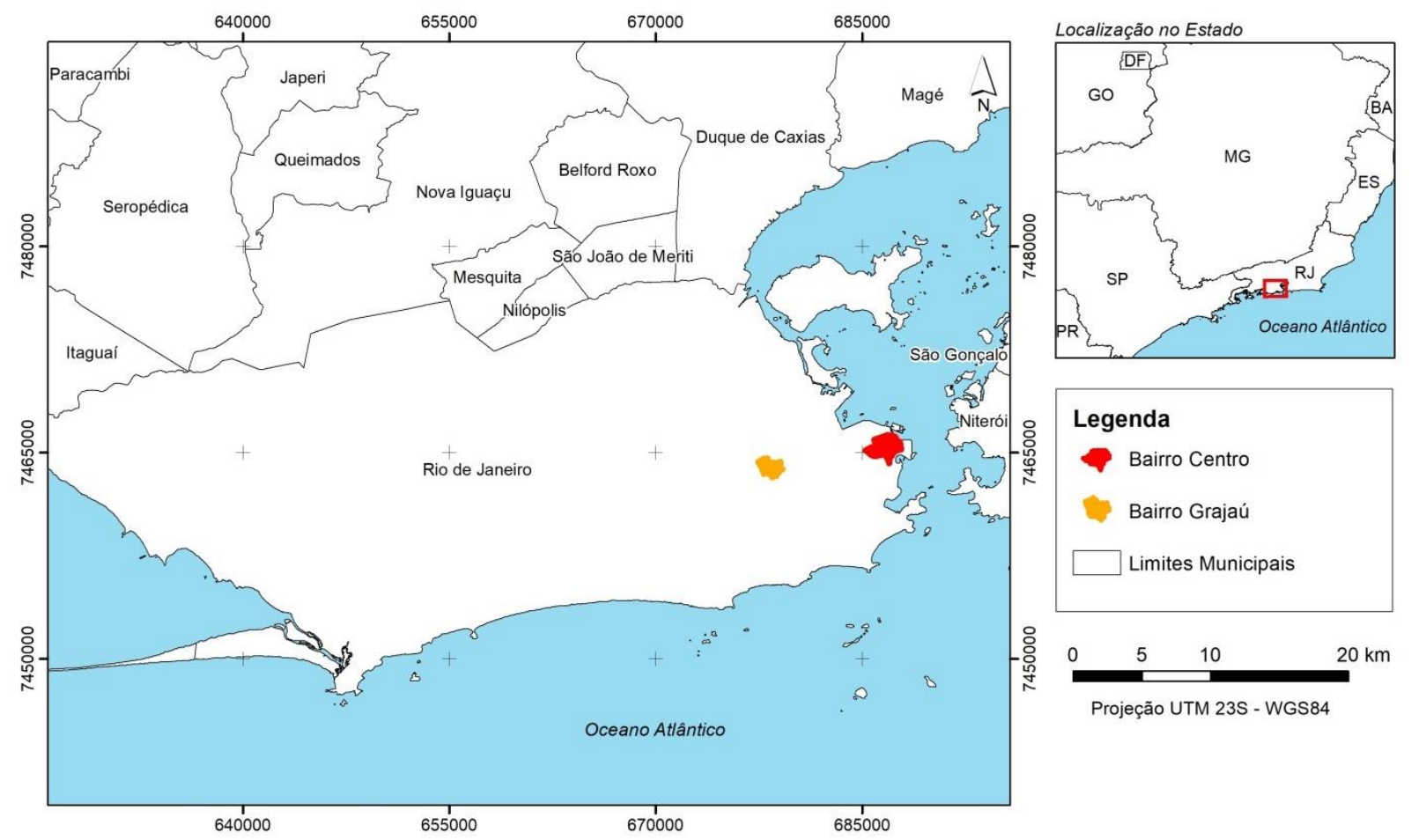

Figura 1. Localização da área de estudo, bairros Centro e Grajaú, no município do Rio de Janeiro (RJ) Figure 1. Location of the study area, district of Centro and Grajaú, in the city of Rio de Janeiro (RJ)

O bairro do Grajaú está situado na zona norte do município do Rio de Janeiro, sendo considerado como local aprazível, com casas jardinadas, ruas largas e arborizadas e clima agradável. Possui 573,9 hectares $\left(5,74 \mathrm{~km}^{2}\right)$ de área, 38.671 habitantes e 15.612 domicílios estando incluído na Região Administrativa IX- Vila Isabel (BAIRROS CARIOCAS, 2018). O bairro Grajaú está no entorno Parque Nacional da Tijuca e compõe o mosaico da vegetação carioca, sendo considerado um importante sítio de interesse ambiental e paisagístico para o município. 
O bairro Centro embora possua prédios residenciais, predomina o uso comercial e turístico; com a maior concentração de edifícios financeiros e de escritórios da cidade. Naturalmente, é uma grande área plana com alguns morros baixos, seu litoral original não existe mais, devido a diversos e graduais aterros, principalmente para a modelação atual do Porto do Rio de Janeiro. O Centro ocupa área de 572,3 hectares $\left(5,72 \mathrm{~km}^{2}\right)$, tem uma população de 41.142 habitantes e faz limite com os bairros Catumbi, Cidade Nova, Gamboa, Glória, Lapa, Santa Teresa, Saúde e Santo Cristo (BAIRROS CARIOCAS, 2018).

A coleta dos dados foi realizada em campo utilizando o aplicativo Collector (desenvolvido pela ESRI e disponível no Play Store) em aparelho de celular Lenovo Vibe K5, onde os atributos foram inseridos em campos específicos já previamente cadastrados, sendo a localização georreferenciada, sem a necessidade de fichas de papel. A mesma metodologia foi utilizada nas duas coletas de dados, porém as informações do trabalho de Silva et al. (2016) foram retiradas diretamente de seu trabalho, no qual em 2015 utilizou o mesmo aplicativo para coleta dos dados. Os dados foram exportados para uma planilha de Excel, para que fosse possível o processamento e análise. Os atributos foram parametrizados da seguinte forma:

a) Tipo de via - beco, travessa, rua, avenida, estrada, praça e largo;

b) Nome vulgar - nome popular atribuído à árvore;

c) Espécie - para atualização dos binômios específicos foi utilizada as informações disponíveis no site do Missouri Botanical Garden (MOBOT, 2018). A classificação adotada foi APG III (THE ANGIOSPERM PHYLOGENY GROUP, 2009);

d) Presença ou não de cancro - protuberâncias em partes do tronco, galhos do vegetal em que a casca ou cambio encontram-se mortos;

e) Presença ou não de cavidade aparente $\geq 30 \%$ da circunferência à altura do peito (CAP) da árvore. Mediu-se o CAP, e quando presente, mediu-se a largura da cavidade;

f) Equilíbrio - avaliou-se se a copa estava equilibrada ou desequilibrada (maior quantidade de copa de um lado da árvore, com potencial risco de favorecer a queda);

g) Danos das raízes - classificou-se o sistema radicular em relação a presença ou ausência de danos às calçadas, danos superficiais (pequenas rachaduras na calçada) e danos das raízes (levantamento de calçadas);

h) Conflitos com a rede de distribuição de energia elétrica - estimou-se a altura dos indivíduos arbóreos com base na altura das construções ( 1 andar $=3 \mathrm{~m}$; e andares $=6 \mathrm{~m} ; \ldots, 8$ andares $=24 \mathrm{~m}$ ) e se havia interceptação ou não na rede;

i) Conflitos com pedestres - dificuldade ou não à passagem de pedestres;

j) Conflitos com fachada - conflito(s) com fachada de casas residenciais ou prédios comerciais. 
Além disso, com o auxílio de uma fita métrica foi mensurada a circunferência a 1,30 m do solo (CAP). Sendo esta necessária para a avaliação de lesões no tronco das árvores. Já os dados de altura foram coletados através de estimativa pelos objetos urbanos, tais como os postes que se encontravam próximos aos vegetais em estudo. O censo do bairro Grajaú ficou restrito apenas às vias públicas (ruas), não sendo considerada nesse estudo a arborização das praças. Todas as árvores foram divididas em três classes de alturas de acordo com o trabalho de Silva et al. (2016): $\leq 6,0 \mathrm{~m} ;>6,0$ e $\leq 9,4 \mathrm{~m} ;>9,4 \mathrm{~m}$, onde correspondem respectivamente, abaixo da rede de energia, entre a rede de baixa e média tensão e acima da rede de média tensão. Foram avaliados por classes de altura o número de indivíduos, a frequência e os conflitos com rede, fachada e pedestres associados a estas árvores.

Os dados espaciais das espécies e os mapas foram processados e elaborados, respectivamente, através do software ArcGIS 10.2, a fim de avaliar visualmente a ocorrência espacial das espécies, suas condições atuais e selecionar os principais atributos. As principais ferramentas utilizadas foram as seguintes: "Select by Attribute", a qual selecionou os atributos do banco de dados coletados através da linguagem SQL (Structured Query Language), que permite uma busca estruturada através de equações simples; "Statistics..." que possibilita listar estatísticas básicas do banco de dados; além das ferramentas para elaboração dos Layouts incluindo a simbolização da legenda e definição de projeções, padronizada como Universal Transversa de Mercator 23K Datum WGS84.

\section{RESULTADOS E DISCUSSÃO}

O censo das vias públicas do bairro do Grajaú no Rio de Janeiro, Rio de Janeiro, totalizou 2.719 árvores de 34 famílias, 71 gêneros e 82 espécies diferentes, sendo 52 espécies classificadas como exóticas e 29 como nativas do Brasil (LORENZI, 1992; LORENZI et. al., 2003). Silva et al. (2016) somente para o bairro Centro, na mesma cidade encontraram 2.693 indivíduos arbóreos distribuídos em 50 espécies. A diversidade de espécies encontradas no bairro Grajaú foi superior ao bairro Centro. Comparando os resultados desse levantamento com trabalhos realizados em outros municípios brasileiros, observou-se que a diversidade é superior aos demais locais, como por exemplo, no estudo de Lima et al. (2007), que no levantamento realizado sistematicamente em $60 \%$ da área urbana do município de Bandeirantes $\left(12 \mathrm{~km}^{2}\right.$ de perímetro urbano), Paraná, encontraram 2.144 árvores distribuídas em 36 espécies, e no levantamento de Mariano e Pereira (2013) no bairro do Parque do Trevo em Ituverava, São Paulo, que encontraram 466 indivíduos distribuídos em 43 espécies. 
As dez espécies de maior frequência encontrada nas vias públicas no bairro Grajaú representaram $73,0 \%$ do total de indivíduos, enquanto que no bairro Centro 83,0\% (Tabela 1). Entre as dez espécies com maiores frequências, seis espécies são comuns em ambos os bairros (Licania tomentosa (Benth.) Fritsch, Handroanthus sp., Cassia sp., Pachira aquatica Aubl., Ficus sp. e Terminalia catappa L.) (Tabela 1), destacando-se Licania tomentosa, a espécie mais frequente no Centro e segunda no Grajaú. Pelo mapa produzido (Figura 2) é possível constatar que as dez árvores mais frequentes no bairro Grajaú estão presentes na maioria das ruas do bairro, no entanto as espécies tamarindeiro (Tamarindus indica L.) e oiti (Licania tomentosa) apresentam-se concentradas em apenas algumas ruas e avenidas.

Tabela 1. Espécies de maior ocorrência nas ruas dos bairros Grajaú e Centro, na cidade do Rio de Janeiro Table 1. Species of greater occurrence in the streets of Grajaú and Centro neighborhoods, in the city of Rio de Janeiro

\begin{tabular}{|c|c|c|c|c|}
\hline \multirow[t]{3}{*}{ Nome vulgar } & Espécie & $\mathrm{Ni}$ & $\mathrm{F}$ & FA \\
\hline & Grajáu & - & \multicolumn{2}{|c|}{$\%$} \\
\hline & Terminalia catappa L. & 429 & 15,7 & 15,7 \\
\hline Oiti & Licania tomentosa (Benth.) Fritsch & 291 & 10,7 & 26,4 \\
\hline Flamboyant & Delonix regia (Bojer ex Hook.) Raf. & 256 & 9,4 & 35,7 \\
\hline Tamarindeiro & Tamarindus indica $\mathrm{L}$. & 248 & 9,1 & 44,8 \\
\hline Ipê & Handroanthus sp & 170 & 6,2 & 51,0 \\
\hline Munguba & Pachira aquatica Aubl. & 160 & 5,9 & 56,9 \\
\hline Cassia-amarela & Senna siamea (Lam.) H.S. Irwin \& Barneby & 153 & 5,6 & 62,5 \\
\hline Ficus & Ficus $s p^{*}$ & 129 & 4,7 & 67,3 \\
\hline Sibipiruna & Caesalpinia pluviosa var. peltophoroides (Benth.) G.P.Lewis & 89 & 3,3 & 70,5 \\
\hline Jasmim-manga & Plumeria rubra L. Var. Alba & 67 & 2,5 & 73,0 \\
\hline Total & & 1992 & 73,0 & - \\
\hline \multicolumn{5}{|c|}{ Centro** } \\
\hline Oiti & Licania tomentosa (Benth.) Fritsch & 1170 & 43,4 & 43,4 \\
\hline Ipê & Handroanthus sp & 320 & 11,9 & 55,3 \\
\hline Palmeira & Arecaceae & 126 & 4,7 & 60,0 \\
\hline Palmeira-imperial & Roystonea oleracea (Jacq.) & 118 & 4,4 & 64,4 \\
\hline Cassia-amarela & Senna siamea (Lam.) H.S. Irwin \& Barneby & 114 & 4,2 & 68,6 \\
\hline Munguba & Pachira aquatica Aubl. & 104 & 3,9 & 72,5 \\
\hline Ficus-microcarpa & Ficus microcarpa L. & 92 & 3,4 & 75,9 \\
\hline Ficus & Ficus sp & 81 & 3,0 & 78,9 \\
\hline Amendoeira & Terminalia catappa L. & 76 & 2,8 & 81,7 \\
\hline Jambeiro & Syzygium malaccense (L.) Merr \& L.M. Perry & 36 & 1,3 & 83,1 \\
\hline Total & & 2237 & 83,0 & - \\
\hline
\end{tabular}




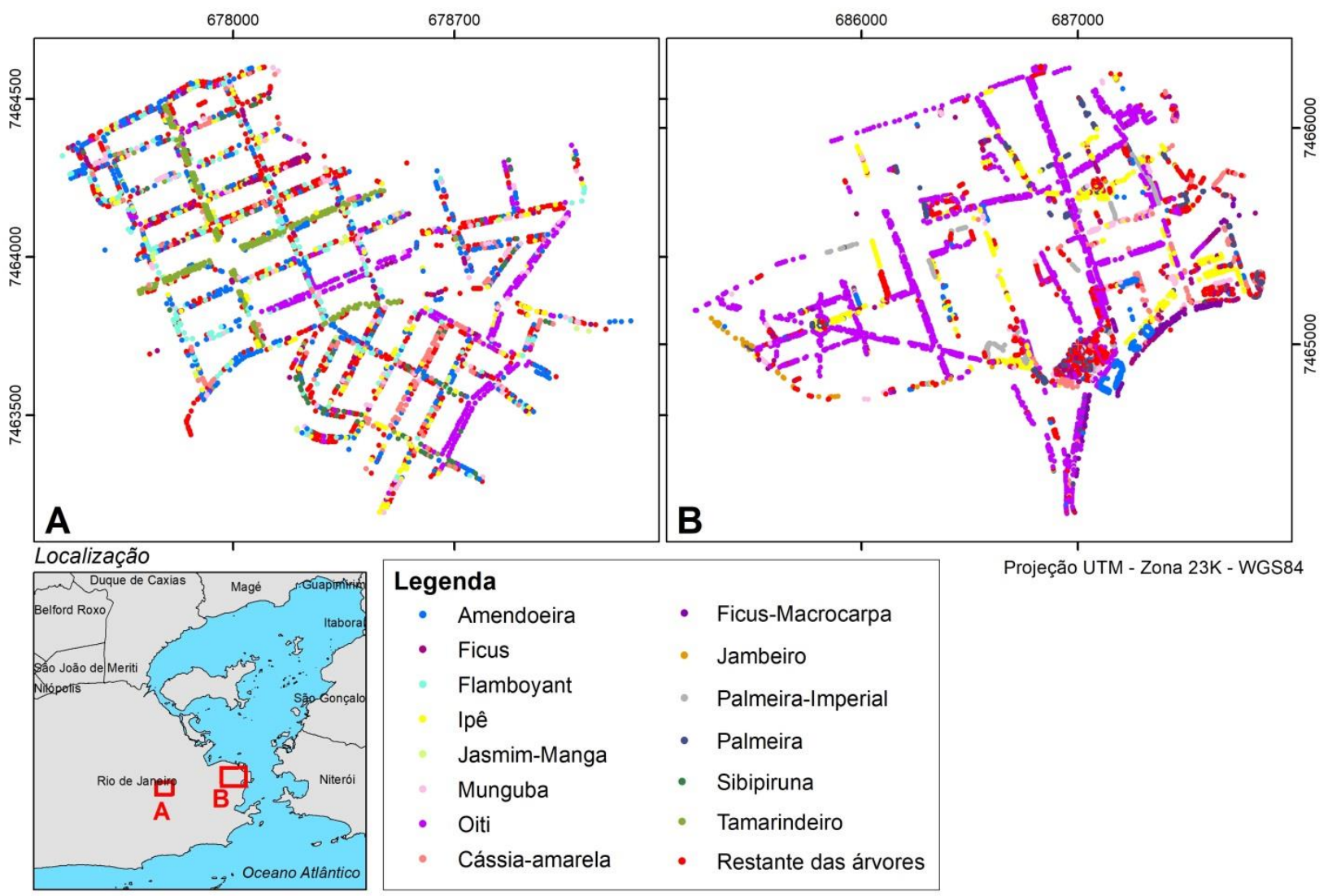

Figura 2. Distribuição espacial das espécies de maior ocorrência no bairro Grajaú (A) e no Centro (B) da cidade do Rio de Janeiro, RJ

Figure 2. Spatial distribution of the most frequent species in Grajaú(A) and in Centro (B) neighborhoods of the city of Rio de Janeiro, RJ

Redin et al. (2010) e Milano (1984) recomendam que a frequência de uma única espécie na arborização urbana não deve ultrapassar a 15\%, de modo a garantir as condições fitossanitárias das árvores, pois um número superior a 15\% pode colocar em risco um grande número de vegetais. No presente estudo, tanto o bairro Grajaú como o Centro, houve espécies acima deste valor (Tabela 1). Considerando o parâmetro de Redin et al. (2010) e Milano (1984), a amendoeira (Terminalia catappa) no Grajaú (15,7\%) e o oiti (Licania tomentosa) no Centro $(43,4 \%)$ estão acima do limite de $15 \%$.

Assim como no presente estudo, o oiti (Licania tomentosa) é uma espécie frequentemente encontrada na arborização urbana em vários municípios do país. Pires et al. (2010) avaliando a arborização do município de Goiandira, Goiás, encontrou frequência de $20,1 \%$ de oiti, sendo uma das espécies de maior ocorrência. A presença dessa espécie no ambiente urbano se deve ao fato de que esta é nativa da Mata Atlântica, bem adaptada ao clima, apresenta copa ampla e farta, fornecendo sombreamento durante todo o ano (LORENZI, 1992).

Melo et al. (2007) constataram que a amendoeira apresentou frequência de 6,71\%, sendo a terceira espécie mais comum no estudo realizado no bairro de Bivar Olinto, em Patos, 
Paraíba. A presença da amendoeira na arborização urbana se deve à sua copa ser densa e ampla, possibilitando grande área sombreada (LORENZI et al., 2003). Outro problema dessa espécie é com relação as folhas que são grandes e coriáceas, entupindo bueiros e sujando as ruas quando as árvores perdem grande parte de suas folhas (de agosto a novembro). A copa ampla de forma horizontal também não se enquadra para o seu plantio em locais que estejam próximos a residências, pois pode promover conflitos com a rede elétrica, com fachadas e telhado de construções. Quando o manejo de poda não ocorre de forma satisfatória para a população, pode gerar insatisfação por parte dos moradores. A Resolução SMAC no 492 de 05 de julho de 2011, instituída pelo o Programa Municipal de Controle de Espécies Exóticas Invasoras Vegetais, indica uma lista de espécies que devem ser evitadas para o plantio no município do Rio de Janeiro, a qual não recomenda o plantio da amendoeira. Sendo necessário que o órgão gestor tenha atenção e monitoramento dessas espécies para que não ocorra sua proliferação de forma desordenada, assim como as que já se encontram em idade avançadas, que sejam monitoradas e substituídas aos poucos, de maneira a atender a resolução.

O flamboyant (Delonix regia), árvore muito empregada na arborização urbana por diversos municípios brasileiros, devido a sua copa bem desenvolvida horizontalmente e sua intensa floração, foi a terceira espécie mais frequente no bairro Grajaú $(9,4 \%)$, não estando entre as dez no bairro Centro (Tabela 1). Em diversos estudos (LIMA et al., 2007; BLUM et al., 2008; MELO et al., 2007; PIRES et al. 2010; SOUZA; CINTRA, 2007), a presença do flamboyant é constante na arborização urbana, no entanto os afloramentos de raízes e danos em calçadas e canteiros também são comuns, o que a torna inadequada para vias públicas.

O tamarindeiro ( Tamarindus indica), com frequência de 9,1\% no bairro Grajaú, não está entre as dez árvores mais frequente no Centro. Esta espécie exótica também foi encontrada em outros estudos (SOUZA; CINTRA, 2007; BLUM et al., 2008; MELO et al., 2007) porém, com frequência menor que um por cento.

O ipê (Handroanthus sp.) foi a segunda espécie de maior ocorrência no bairro Centro (11,9\%) e quinta no bairro Grajaú (6,2\%) do Rio de Janeiro (Tabela 1). Em trabalho realizado no campus da Universidade Federal de Santa Maria, Gracioli et al. (2011) encontraram o ipê (Handroanthus chrysotrichus) em grande abundância, com frequência de 16,0\% e assim como outros autores alertam para o fato de não ser algo ideal para a arborização urbana, pois superior a este percentual pode facilitar a propagação de pragas por estarem numa quantidade alta em relação as demais espécies.

A munguba (Pachira aquatica) apresentou frequência de 5,9 e 3,9\% nos bairros Grajaú e Centro, respectivamente (Tabela 1). Mariano e Pereira (2013) no estudo realizado no Parque do Trevo na cidade de Ituverava, São Paulo, encontraram a munguba como a terceira mais frequente, totalizando 55 indivíduos. De acordo com Lorenzi et al. (1992) a munguba é uma das 
espécies utilizadas na arborização urbana, pois esta apresenta copa volumosa e farta, de forma a fornecer sombra durante todo o ano, exceto pelos frutos enormes que podem cair e causar acidentes, raramente.

A cassia-amarela (Senna siamea) no Grajaú apresentou frequência de 5,6\%, quantidade maior que a apresentada no Centro, onde a mesma espécie teve frequência de 4,2\% (Tabela 1). Souza e Cintra (2007) também constataram a presença da cassia-amarela na arborização urbana do bairro da Taquara, Rio de Janeiro, com frequência de 2,1\%.

No bairro do Grajaú, a figueira foi analisada por gênero (Ficus sp.), estando dentro do percentual encontrado as seguintes espécies: Ficus benjamina L.; Ficus microcarpa L.; e Ficus elástica Roxb. ex Hornem. Foi encontrada no bairro uma frequência de 4,7\% (Tabela 1), e no bairro Centro, Silva et al. (2016) analisaram o Ficus microcarpa e o Ficus sp., tendo frequência de 3,4 e 3,0\%, respectivamente. A presença da figueira na arborização urbana é bastante comum, como podemos observar nos trabalhos de Nepomuceno et al. (2016) que encontraram a Ficus sp. com frequência de $0,9 \%$ e 3,3\% em duas praças de Caratinga, Minas Gerais; de Lima et al. (2007) onde encontraram a Ficus benjamina com frequência de 6,2\% no município de Bandeirantes, Paraná; de Emer et al. (2014) onde em sua análise quali-quantitativa observaram a presença da Ficus benjamina com frequência de $0,94 \%$ no bairro Jardim Primavera na cidade de Pato Branco, Paraná; e Blum et al. (2008) em Maringá, Paraná, observaram a presença da Ficus benjamina com frequência de 2,4\%.

No bairro do Grajaú 150 árvores (5,5\% do total) apresentaram lesões causadas pelo cancro, e Silva et al. (2016) constataram no Centro, 53 indivíduos (2,0\% do total) com a mesma doença (Tabela 2).

Analisando os indivíduos com a presença cancro, 18 diferentes espécies (22\%) apresentaram ocorrência de cancro no bairro Grajaú e 8 espécies (16\%) no bairro Centro (Tabela 2). Em ambos os bairros a amendoeira (Terminalia catappa) foi a espécie com maior frequência com cancro, 71,3 e 58,5\% no bairro Grajaú e no Centro, respectivamente (Tabela 2), evidenciando ser uma espécie susceptível a essas lesões. Ao comparar os dados encontrados nos trabalhos de Silva et al. (2016) e Sampaio et al. (2010), é possível analisar que o bairro Grajaú apresenta valores bem abaixo, mesmo quando esta frequência é superior a do bairro Centro. É necessário que ocorra um monitoramento das árvores com cancro, pois este é um dos parâmetros associados aos fatores de risco. Também se faz necessário a substituição das árvores que apresentam cancro em situações mais avançadas.

Tabela 2. Ocorrência de cancro nas espécies da arborização urbana das ruas dos bairros Grajaú e Centro, da cidade do Rio de Janeiro, RJ 
Table 2. Occurrence of cancer in species of the urban afforestation of the streets of Grajaú and Centro neighborhoods of Rio de Janeiro city, RJ

\begin{tabular}{|c|c|c|c|}
\hline Nome vulgar & Espécie & $\mathrm{Ni}$ & $A B$ \\
\hline & Grajáu & & $\%$ \\
\hline Amendoeira & Terminalia catappa L. & 107 & 71,3 \\
\hline Cassia-amarela & Senna siamea (Lam.) H.S. Irwin \& Barneby & 12 & 8,0 \\
\hline Flamboyant & Delonix regia (Bojer ex Hook.) Raf. & 8 & 5,3 \\
\hline Munguba & Pachira aquatica Aubl. & 4 & 2,7 \\
\hline Tamarindeiro & Tamarindus indica L. & 3 & 2,0 \\
\hline Sibipiruna & Caesalpinia pluviosa var. peltophoroides (Benth.) G.P.Lewis & 2 & 1,3 \\
\hline Cassia & Cassia sp. & 2 & 1,3 \\
\hline Oiti & Licania tomentosa (Benth.) Fritsch & 2 & 1,3 \\
\hline Albizia & Albizia lebbeck (L.) Benth. & 1 & 0,7 \\
\hline Pata-de-vaca & Bauhinia sp. & 1 & 0,7 \\
\hline Sombreiro & Clitoria fairchildiana R.A. Howard. & 1 & 0,7 \\
\hline Ipê & Handroanthus sp. & 1 & 0,7 \\
\hline Canafistula & Peltophorum dubium (Spreng.) Taub. & 1 & 0,7 \\
\hline Goiabeira & Psidium guajava L. & 1 & 0,7 \\
\hline Espatódea & Spathodea campanulata P. Beauv. & 1 & 0,7 \\
\hline Caja & Spondias mombin L. & 1 & 0,7 \\
\hline Pau-formiga & Triplaris americana $\mathrm{L}$. & 1 & 0,7 \\
\hline $\mathrm{NI}$ & - & 1 & 0,7 \\
\hline \multirow[t]{2}{*}{ Total } & & 150 & 100,0 \\
\hline & Centro* & & \\
\hline Amendoeira & Terminalia catappa L. & 31 & 58,5 \\
\hline Cássica-amarela & Senna siamea (Lam.) H.S. Irwin \& Barneby & 10 & 18,9 \\
\hline Ipê & Handroanthus sp. & 4 & 7,5 \\
\hline Munguba & Pachira aquatica Aubl. & 4 & 7,5 \\
\hline Ficus & Ficus sp. & 1 & 1,9 \\
\hline Leucena & Leucaena leucocephala (Lam.) de Wit & 1 & 1,9 \\
\hline Painera & Ceiba speciosa (A. St.-Hill.) Ravenna & 1 & 1,9 \\
\hline $\mathrm{NI}$ & - & 1 & 1,9 \\
\hline Total geral & & 53 & 100,0 \\
\hline
\end{tabular}

De forma similar, a cassia-amarela (Senna siamea) foi a segunda espécie com maior frequência com cancro, 8,0 e 18,9\%, respectivamente nos bairros Grajaú e Centro (Tabela 2). A cassia-amarela é uma espécie que apresenta facilidade de quebra de galhos, ocasionando lesões que podem vir a resultar em doenças, como o cancro.

Embora a maior diversidade seja desejável para maior proteção das espécies com relação ao ataque de pragas e de doenças, isso não foi verificado no presente estudo, onde o bairro Grajaú com maior diversidade (82 espécies) teve maior ocorrência do cancro do que no Centro (50 espécies).

No bairro do Grajaú foram encontradas 37 árvores (1,4\% do total) com cavidade igual ou superior à 30\% da CAP e Silva et al. (2016) encontraram 235 árvores (8,7\% do total) no Centro (Tabela 3).

Tabela 3. Ocorrência de indivíduos com cavidade igual ou maior a $30 \%$ da circunferência a altura do peito (CAP) nas espécies da arborização urbana das ruas dos bairros Grajaú e Centro, da cidade do Rio de Janeiro, RJ 
Table 3. Occurrence of individuals with a cavity equals or greater than $30 \%$ of the circumference at the breast height in the urban tree species of the streets of Grajaú and Centro neighborhoods, in Rio de Janeiro city, RJ

\begin{tabular}{llcc}
\hline Nome vulgar & Espécie & $\mathrm{Ni}$ & $\mathrm{F}$ \\
\hline Tamarindeiro & \multicolumn{1}{c}{ Grajáu } & $\%$ \\
Não-identificado & Tamarindus indica L. & 26 & 70,3 \\
Cássia-amarela & - & 2 & 5,4 \\
Amendoeira & Senna siamea (Lam.) H.S. Irwin \& Barneby & 2 & 5,4 \\
Sombreiro & Terminalia catappa L. & 2 & 5,4 \\
Flamboyant & Clitoria fairchildiana R.A. Howard. & 1 & 2,7 \\
Ficus & Delonix regia (Bojer ex Hook.) Raf. & 1 & 2,7 \\
Mangueira & Ficus sp. & 1 & 2,7 \\
Munguba & Mangifera indica L. & 1 & 2,7 \\
Total & Pachira aquatica Aubl. & 1 & 2,7 \\
& $-\quad$ Centro* & 37 & 100 \\
Oiti & Licania tomentosa (Benth.) Fritsch & 190 & 80,9 \\
Cássia-amarela & Senna siamea (Lam.) H.S. Irwin \& Barneby & 18 & 7,7 \\
Ficus-macrocarpa & Ficus macrocarpa L. & 17 & 7,2 \\
Palmeira & Arecaceae & 5 & 2,1 \\
Munguba & Pachira aquatica Aubl. & 5 & 2,1 \\
Total & - & 235 & 100 \\
\hline Nota: $\mathrm{Ni}=$ Número de indivíduos; $F(\%)$ & Frequência. ${ }^{*}$ Adaptado de Silva et al. (2016) & &
\end{tabular}

De acordo com a Companhia Energética de Minas Gerais (CEMIG) (2011) a presença de cavidade pode indicar um risco de queda das árvores, pois esta cavidade pode afetar a sustentação da mesma. Segundo a CEMIG (2011), estas cavidades são formadas por meio das mais diversas fontes: podas mal executadas, fogo, resposta a atividades de insetos ou outros animais, depredação ou acidentes. Além disso, doenças como a podridão ocasionadas pela presença de fungos geram o apodrecimento de parte da árvore.

O bairro Centro apresentou maior frequência de árvores com cavidade do que o Grajaú, o que pode ser associado ao Centro concentrar a área comercial da cidade, acarretando em maior trânsito de pessoas e veículos. O monitoramento dessas árvores é importante, bem como é necessário e a substituição das que apresentam índice de cavidade elevado, proposto por Mariano e Pereira (2013).

Ao analisar as árvores com cavidades no bairro Grajaú, percebe-se que o maior percentual se concentra nos tamarindeiros (Tamarindus indica) com frequência de $70,3 \%$. Os tamarindeiros com cancro estavam localizados em sua maior parte, no canteiro central da Avenida Engenheiro Richard e da Avenida Júlio Furtado, são árvores imunes ao corte sendo protegidas pelo Decreto Municipal no. 27.380, de 29 de novembro de 2006 (Figura 2 e 3). Nesse local funciona um estacionamento irregular de veículos, e as cavidades se formaram devido ao impacto mecânico ocasionado pelos para-lamas dos veículos, o que pode ser evidenciado pela altura e formato das cavidades formadas (Figura 3). No bairro Centro, 80,9\% das árvores com cavidade são da espécie oiti (Licania tomentosa), de acordo com Silva et al. (2016). 


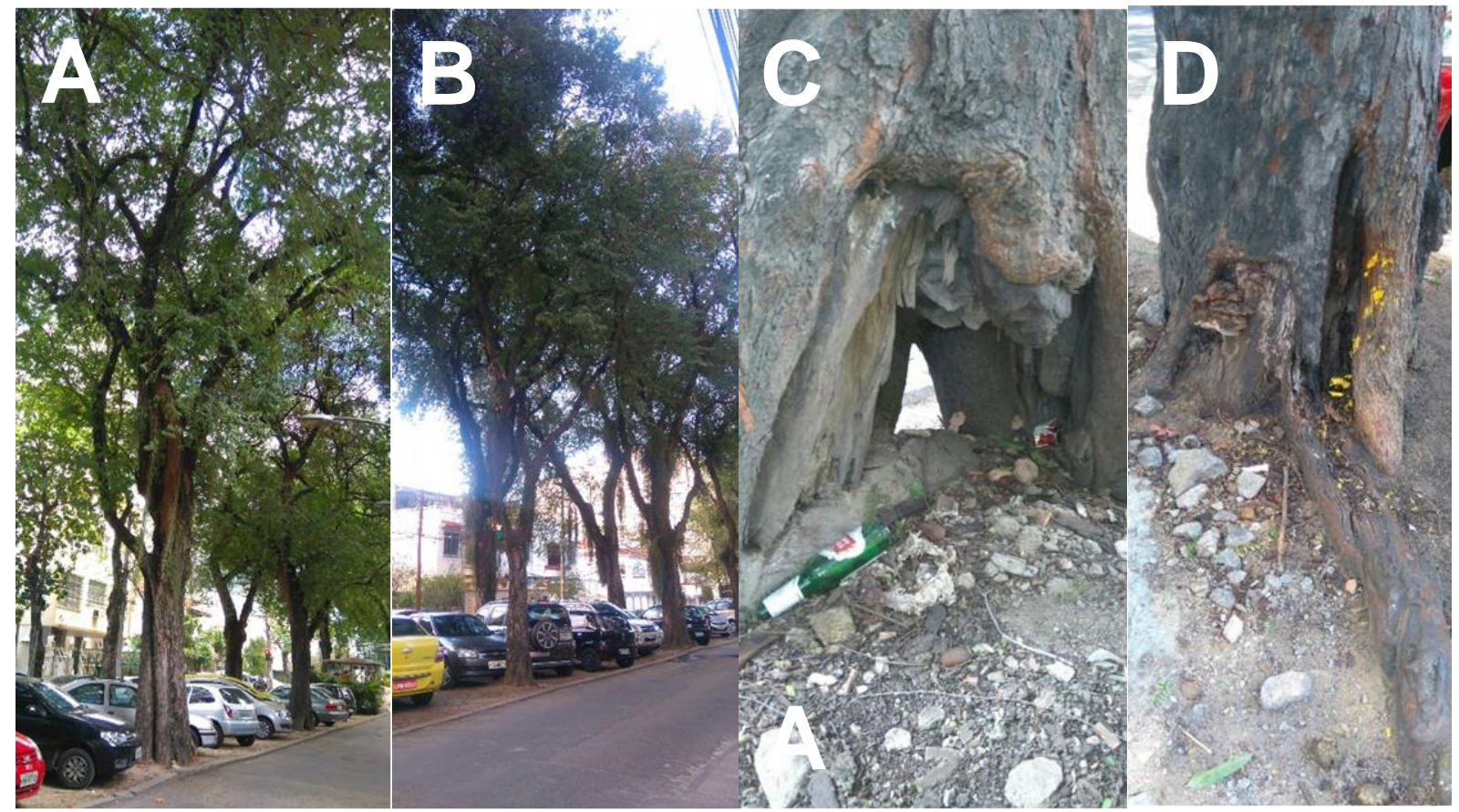

Figura 3. Canteiro central da Avenida Engenheiro Richard na cidade do Rio de Janeiro RJ. A e B: carros estacionados no bairro Grajaú; C e D: cavidades na base dos tamarindeiros

Figure 3. Central island of Engenheiro Richard Avenue in Rio de Janeiro city, RJ. A and B: cars parked in Grajaú neighborhood; $C$ and D: cavities at the base of the tamarindeiros

O bairro Grajaú apresentou 37 indivíduos com presença de cavidade, onde a maioria se encontra nos tamarindeiros, com 27 indivíduos. É possível perceber que outros autores também apontaram a presença de cavidades nas árvores urbanas, Nepomuceno et al. (2016) na cidade de Caratinga, Minas Gerais, observaram a frequência de $20,3 \%$ de árvores com alguma cavidade no tronco. Oliveira et al. (2016) na região oeste de Belo Horizonte, Minas Gerais, observaram frequência de $1,62 \%$ de Ligustrum japonicum Thunb. com cavidade na base, afirmando que a espécie é vulnerável a formação de cavidades na base e no tronco da árvore se assemelhando aos dados obtidos no bairro do Grajaú onde as árvores também apresentam cavidades na base (Figura 3).

Observa-se que a distribuição espacial das árvores com cancro e, ou cavidade não apresenta um padrão específico (Figura 4). Há uma tendência de mais indivíduos com cavidade nas avenidas acima citadas em função da presença do tamarindeiro. 


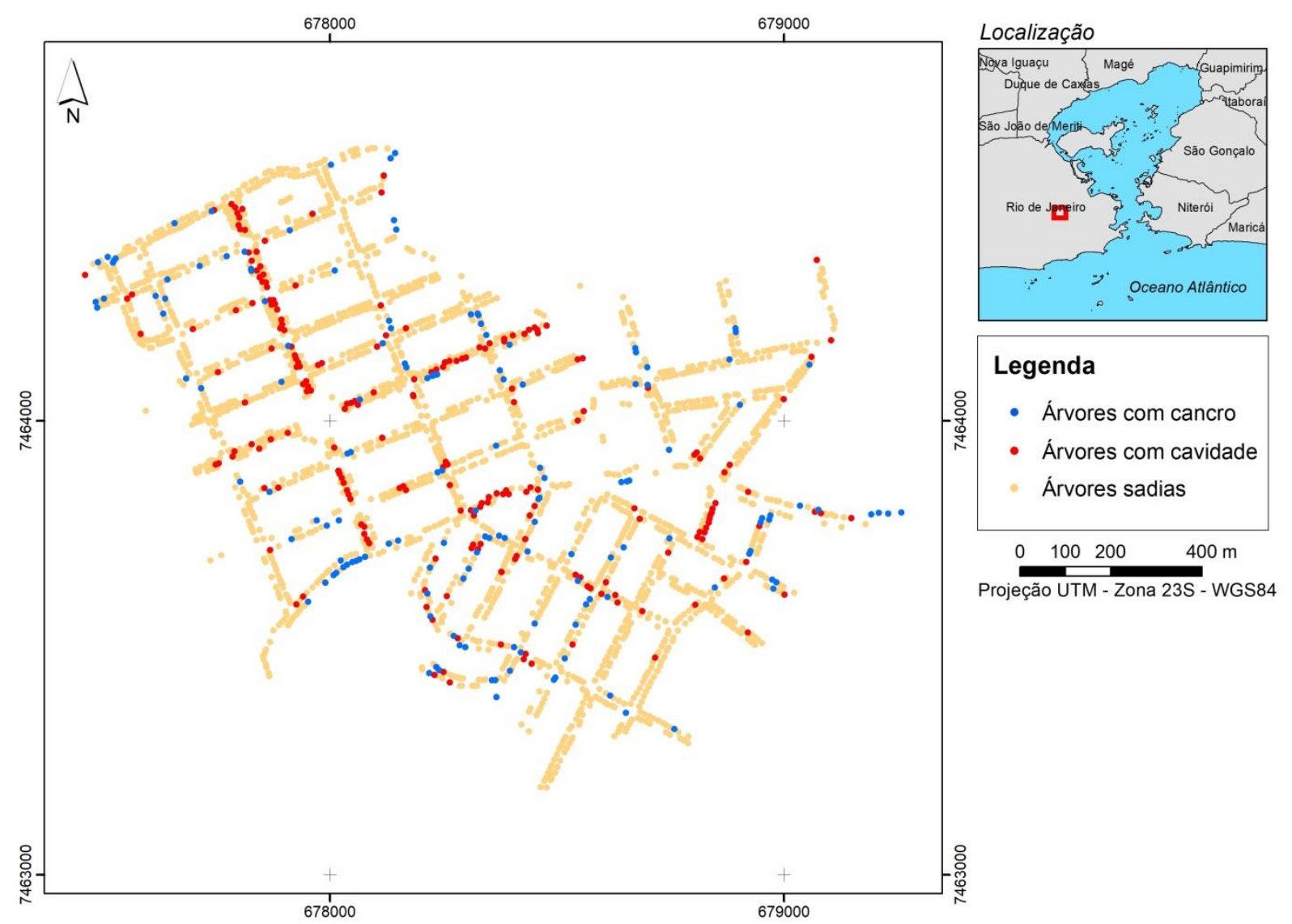

Figura 4. Localização das árvores com cancro, com cavidades e sadias no bairro Grajaú, cidade do Rio de Janeiro, RJ, apontando aquelas com cancro e ou cavidade

Figure 4. Location of trees with cancer, cavities and healthy in Grajaú neighborhood, Rio de Janeiro city, $\mathrm{RJ}$, pointing out those with cancer and/or cavity

Os indivíduos arbóreos com copa desequilibrada e algum dano aparente na raiz no bairro Grajaú totalizaram 89 árvores e no Centro 24 árvores (Tabela 4). No bairro Grajaú duas espécies se destacam, a amendoeira (Terminalia catappa) e o flamboyant (Delonix regia), sendo essas espécies a primeira e a terceira mais frequente nesse bairro. No Centro, de forma diferente o problema se concentra nas figueiras (Ficus microcarpa) e no oiti (Licania tomentosa), esta última a mais frequente.

No bairro Grajaú o índice de árvores com copas desequilibradas e com danos à raiz foi baixo (3,3\% do total de árvores) e muito se deve pela escolha equivocada das espécies amendoeiras (Terminalia catappa) e flamboyant (Delonix regia) que apresentam estrutura de copa semelhantes, bem distribuída de forma horizontal (LORENZI et al., 2003). Essa estrutura de copa pode ocasionar conflitos com telhados e fachadas de residências. Além disso, ambas apresentam raízes superficiais bem desenvolvidas, causando danos às calçadas, muros e até mesmo bloqueio da passagem de pedestres no passeio público. Essa constatação dos danos encontrados nas raízes, assim como na copa, são provavelmente promovidas pelos próprios 
moradores na tentativa de reduzir o conflito existente. No bairro Centro esse índice foi ainda menor ( $0,9 \%$ do total) e se concentrou no oiti (Licania tomentosa), a espécie mais frequente no bairro e no Ficus macrocarpa, espécie com sistema radicular superficial e causador de muitos danos.

Tabela 4. Árvores com copa desequilibrada e danos da raiz nas espécies da arborização urbana das ruas dos bairros Grajaú e Centro, da cidade do Rio de Janeiro, RJ.

Table 4. Trees with unbalanced canopy and root damage in the urban afforestation species of the streets of Grajaú and Centro neighborhoods, in Rio de Janeiro city, RJ.

\begin{tabular}{|c|c|c|c|}
\hline Nome vulgar & Espécie & $\mathrm{Ni}$ & $\mathrm{F}$ \\
\hline & Grajáu & & $\%$ \\
\hline Amendoeira & Terminalia catappa $L$. & 21 & 23,6 \\
\hline Flamboyant & Delonix regia (Bojer ex Hook.) Raf. & 20 & 22,5 \\
\hline Sibipiruna & Caesalpinia pluviosa var. peltophoroides (Benth.) G.P.Lewis & 6 & 6,7 \\
\hline Ficus & Ficus sp. & 6 & 6,7 \\
\hline Tamarindeiro & Tamarindus indica $\mathrm{L}$. & 6 & 6,7 \\
\hline Cássia-amarela & Senna siamea (Lam.) H.S. Irwin \& Barneby & 5 & 5,6 \\
\hline Pata-de-Vaca & Bauhinia sp & 4 & 4,5 \\
\hline Munguba & Pachira aquatica Aubl. & 4 & 4,5 \\
\hline Oiti & Licania tomentosa (Benth.) Fritsch & 4 & 4,5 \\
\hline Goiabeira & Psidium guajava L. & 3 & 3,4 \\
\hline Cassia & Cassia sp. & 2 & 2,2 \\
\hline Aroeira & Schinus terebinthifolius Raddi & 2 & 2,2 \\
\hline Albizia & Albizia lebbeck (L.) Benth. & 1 & 1,1 \\
\hline Flamboyant-mirim & Caesalpinia pulcherrima & 1 & 1,1 \\
\hline Extremosa & Lagerstroemia indica & 1 & 1,1 \\
\hline Mangueira & Mangifera indica L. & 1 & 1,1 \\
\hline $\mathrm{NI}$ & - & 1 & 1,1 \\
\hline Tipuana & Tipuana tipu & 1 & 1,1 \\
\hline Total & & 89 & 100 \\
\hline \multicolumn{4}{|c|}{ Centro* } \\
\hline Ficus-macrocarpa & Ficus macrocarpa L. & 10 & 41,7 \\
\hline Oiti & Licania tomentosa (Benth.) Fritsch & 7 & 29,2 \\
\hline Cássia-amarela & Senna siamea (Lam.) H.S. Irwin \& Bameby & 3 & 12,5 \\
\hline Casuarina & Casuarina equisetifolia L. & 1 & 4,2 \\
\hline Ficus & Ficus sp. & 1 & 4,2 \\
\hline Figueira-benjamim & Ficus benjamina L. & 1 & 4,2 \\
\hline Munguba & Pachira aquatica Aubl. & 1 & 4,2 \\
\hline Total & - & 24 & 100 \\
\hline
\end{tabular}

Nota: $\mathrm{Ni}$ = Número de indivíduos; $\mathrm{F}(\%)$ = Frequência; $\mathrm{NI}$ - Não Identificada. *Adaptado de Silva et al. (2016)

A maior parte das árvores do bairro Grajaú se apresenta com a copa medianamente equilibrada, no entanto existe uma parcela dessas árvores em que a copa está desequilibrada (Figura 5). Pereira et al. (2011) afirmaram que um dos fatores que influenciam diretamente a estabilidade das árvores é o tipo que poda que sofrem, onde podas de rebaixamento ou em forma de "U", quando realizadas de forma equivocada, podem descolar o centro de massa da árvore para alguma lateral, causando desequilíbrio e aumentando o risco de queda, sendo mais acentuado caso essas árvores apresentem problemas como cavidades em sua base. 


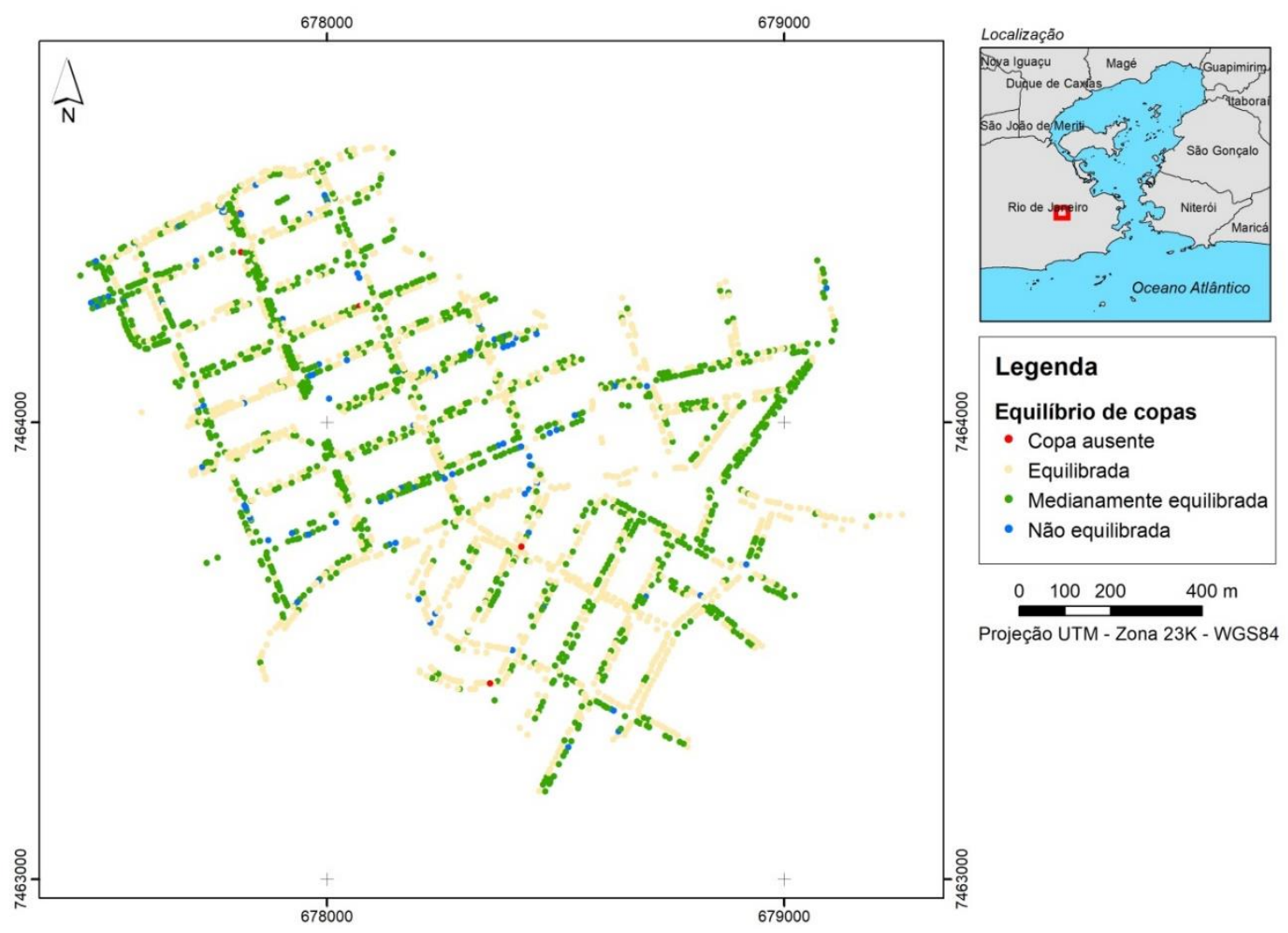

Figura 5. Distribuição espacial das árvores indicando aquelas com copa ausente, equilibrada, medianamente equilibrada e não equilibrada no bairro Grajaú na cidade do Rio de Janeiro, RJ

Figure 5. Spatial distribution of the trees indicating those with absent, balanced, moderately balanced and unbalanced canopy in Grajaú neighborhood in Rio de Janeiro city, RJ

A divisão das árvores por classe de altura (Tabela 5) mostra que a maior parte possui altura superior a 9,4 m no bairro Grajaú $(56,9 \%)$ e no Centro $(70,8 \%)$.

Tabela 5. Número de indivíduos divididos em três classes de altura e a frequência dos indivíduos que conflitam com a rede de distribuição de energia, fachada e pedestre dentro de cada classe nas espécies da arborização urbana das ruas dos bairros Grajaú e Centro, da cidade do Rio de Janeiro, RJ

Table 5. Number of individuals divided into three height classes and the frequency of individuals conflicting with the energy network, facade and pedestrian within each class in the urban tree species of the streets of Grajaú and Centro neighborhood of Rio de Janeiro city, RJ

\begin{tabular}{ccccc}
\hline Classe de altura $(\mathrm{m})$ & $\mathrm{Ni}(\%)$ & \multicolumn{1}{c}{ Rede $(\%)$} & \multicolumn{1}{c}{ Fachada $(\%)$} & Pedestre $(\%)$ \\
\hline $\mathrm{H} \leq 6,0$ & $691(25,4)$ & $43(1,6)$ & $29(1,1)$ & $80(2,9)$ \\
$6,0<\mathrm{H} \leq 9,4$ & $482(17,7)$ & $85(3,1)$ & $122(4,5)$ & $24(0,9)$ \\
$\mathrm{H} \geq 9,4$ & $1546(56,9)$ & $161(5,9)$ & $635(23,4)$ & $35(1,3)$ \\
Total Geral & 2719 & $289(10,6)$ & $786(29,0)$ & $139(5,1)$ \\
& & \multicolumn{2}{c}{ Centro*$^{*}$} & \\
$\mathrm{H} \leq 6,0$ & $659(17,0)$ & $5(0,8)$ & $17(2,6)$ & $56(8,5)$ \\
$6,0<\mathrm{H} \leq 9,4$ & $474(12,2)$ & $9(1,9)$ & $46(9,7)$ & $53(11,2)$ \\
$\mathrm{H} \geq 9,4$ & $2741(70,8)$ & $72(2,6)$ & $722(26,3)$ & $82(3,0)$ \\
Total Geral & 3874 & $86(2,2)$ & $785(20,3)$ & $191(4,9)$ \\
\hline Nota: $\mathrm{Ni}=$ Número de indivíduos; $\mathrm{F}(\%)=$ Frequência. ${ }^{*}$ Adaptado de Silva et al (2016) - dados das ruas e das praças do bairro Centro
\end{tabular}


De acordo com os resultados encontrados na literatura, observa-se que grande parte da arborização urbana das cidades não se atentou ao potencial de conflito entre rede elétrica e árvores, onde órgãos públicos e os moradores plantam indivíduos de porte acima do recomendado frequentemente abaixo das redes elétricas. É necessário que o planejamento seja realizado de forma criteriosa para que problemas com a fiação sejam evitados. Nesse sentido o uso de ferramentas de geoprocessamento funciona como direcionamento do planejamento, orientando as redes elétricas e os possíveis locais de plantio.

Nos conflitos com a rede elétrica, observa-se que tanto no bairro Grajaú como no Centro a maior frequência de árvores conflitantes são aquelas com altura acima de $9,4 \mathrm{~m}$ de altura (5,9\% no Grajaú e 2,6\% no Centro). De acordo com Silva et al. (2016) a baixa taxa de árvores em conflito com rede encontradas no bairro Centro (86 árvores) é devido ao uso em sua maioria da rede subterrânea de energia. Já no bairro Grajaú houve 289 árvores em conflitos com a rede de energia, pelo fato de se tratar de bairro residencial e sem a presença de rede subterrânea. Nepomuceno et al. (2016) no estudo na cidade de Caratinga, Minas Gerais, afirmaram que a maioria dos guias de arborização recomendam que a rede elétrica e a arborização urbana ocupem lados diferentes. $O$ autor também recomenda o uso de espécies de pequeno porte sob redes elétricas a fim de evitar eventuais conflitos. Em estudo realizado por Lima et al. (2007) em Bandeirantes, Minas Gerais, foi possível perceber que conflitos com rede elétrica e telefônica apresentam problemas semelhantes aos encontrados no bairro do Grajaú, sendo a fiação e árvores situadas do mesmo lado da calçada, com um total de $31,6 \%$ de árvores em conflitos em Bandeirantes.

Referente aos conflitos das árvores com as fachadas foi observado que no bairro Grajaú a maior frequência encontrada foi nas árvores com altura acima de 9,4 m (23,4\% de todas as árvores). De forma semelhante, o bairro Centro também apresentou a maior frequência nas árvores de altura acima de 9,4 m (26,3\%). Lima et al. (2007) na cidade de Bandeirantes, Minas Gerais, observaram que 3,1\% das árvores analisadas se encontravam em conflito com a fachada dos estabelecimentos, valor bem abaixo do encontrado no presente levantamento.

A análise dos dados permite inferir que as árvores do bairro Grajaú comparadas com as do bairro Centro apresentaram maior quantidade de conflitos e problemas fitossanitários. É possível que estes problemas sejam mais frequentes no bairro do Grajaú por ser uma área residencial e com maior dificuldade para medidas de manejo. $O$ serviço de atendimento a solicitações de manejo arbóreo da prefeitura do Rio de Janeiro em muitas vezes não consegue executar a atividade devido a não concordância da população com a poda; carros estacionados abaixo das árvores; redes de alta tensão impossibilitando o manejo; entre outros, fazendo com que o manejo se torne menos eficiente, principalmente nos bairros de caráter mais residencial como o Grajaú. 
As árvores do bairro Centro apresentaram maior frequência de cavidades, o que pode indicar maior vandalismo em função do fluxo de pessoas e obras (SILVA et al., 2016). Porém, por se tratar de área em que apresenta maior visibilidade, normalmente recebe uma atenção maior quanto às suas solicitações, logo a qualidade no atendido e manejo é realizada para evitar maiores problemas de reclamação para o órgão competente. A rede elétrica no bairro Centro é subterrânea, o que faz que o manejo seja mais fácil e consequentemente diminua a frequência de conflitos.

O planejamento realizado utilizando as ferramentas de georeferenciamento possibilita maior visualização dos problemas e associação do mesmo, dando possibilidades de maior planejamento e eliminação dos problemas para o engenheiro responsável.

\section{CONCLUSÕES}

A arborização das ruas do bairro Grajaú comparada a do Centro apresentou maior número de árvores e diversidade de espécies, embora a maioria seja de espécies exóticas. A amendoeira (Terminalia catappa) foi a espécie com maior frequência no bairro do Grajaú, sendo também a de maior frequência com cancro, copa desequilibrada e danos à raiz. O oiti (Licania tomentosa) foi a mais frequente no Centro e a segunda no Grajaú, sendo uma espécie com característica adequada e muito frequente na arborização de ruas de cidades do país.

Em ambos os bairros os números de árvores com cancro, cavidades e conflitos foram relativamente baixo quando comparados a arborização urbana de outras cidades, o que indica ocorrer um bom manejo arbóreo. Também foi observado que esses problemas estão concentrados em poucas espécies.

O tamarindeiro ( Tamarindus indica) apresentou uma distribuição espacial predominante em duas avenidas e também concentrou a ocorrência de cavidades no bairro Grajaú. Em função da aglomeração dos tamarindeiros nas duas avenidas no bairro Grajaú, é necessária a emissão de autorização do órgão competente para a realização do manejo. Além disso, é prioritário que essas árvores tenham acompanhamento criterioso e medidas para evitar o risco de queda.

Com a produção dos mapas e o auxílio do geoprocessamento foi possível visualizar com facilidade a distribuição e onde estão localizados os problemas, auxiliando assim no planejamento e no manejo da arborização urbana nos bairros estudados.

\section{REFERÊNCIAS}


ALVARES, C.A.; STAPE, J.L.; SENTELHAS, P.C.; GONÇALVES, J.L.M.; SPAROVEK, G. Köppen's climate classification map for Brazil. Meteorologische Zeitschrift, Stuttgart, v.22, n.6, p.711-728, 2013.

BAIRROS CARIOCAS, Prefeitura Municipal do Rio de Janeiro. 2018. Disponível em <http://pcrj.maps.arcgis.com/apps/MapJournal/index.html?appid=096ae1e5497145838ca64191 be66f3e3>. Acesso em 11 mar. 2018.

BLUM, C.T.; BORGO, M.; SAMPAIO, A.C.F. Espécies exóticas invasoras na arborização de vias públicas de Maringá-PR. Revista da Sociedade Brasileira de Arborização Urbana, Piracicaba, v.3, n.2, p.78-97, 2008.

COMPANHIA DE ENERGIA ELETRICA DE MINAS GERAIS - CEMIG, Manual de Arborização. Belo Horizonte, Fundação Biodiversitas, p.112, 2011.

EMER, A.A.; CADORIN, D.A.; MELLO, N.A. Avaliação quali-quantitativa da arborização do bairro Jardim Primavera na cidade de Pato Branco - PR. Revista Científica ANAP Brasil, Tupã, v. 7, p. 19-32, 2014.

FARIA, J.L.G.; MONTEIRO, E.A.; FISCH, S.T.V. Arborização de vias públicas do município de Jacareí - SP. Revista da Sociedade Brasileira de Arborização Urbana. Piracicaba, v. 2, n. 4, p. 20-33, 2007.

GRACIOLI, C.R.; BOHNER, T.O.L.; REDIN, C.G.; SILVA, D.T. Arborização do Campus da Universidade Federal de Santa Maria e conscientização da comunidade acadêmica. Revista Eletrônica do Curso de Especialização em Educação Ambiental da UFSM, Santa Maria, v.3, n.3, p. 421-429, 2011.

LIMA NETO, E.M.; BIONDI, D.; ARAKI, H.; BOBROWSKI, R. Fotografias aéreas para mensuração da área de copa das árvores de ruas de Curitiba - PR. Revista Floresta, Curitiba, v.42, p.577-588, 2012.

LIMA, C.B.; BELLETTINI, N.M.T.; SILVA, A.S.; JANANI, J.K.; AMADOR, T.S.; VIEIRA, M.A.V.; CHEIRUBIM, A.P. Descrição das árvores encontradas nas ruas de Bandeirantes-PR. Revista Brasileira de Biociências, Porto Alegre, v.5, supl.1, p.609-611, 2007.

LORENZI, H. Árvores brasileiras - manual de identificação e cultivo de plantas arbóreas nativas no Brasil. Nova Odessa: Instituto Plantarum de Estudos da Flora, 1992. 360p.

LORENZI, H.; SOUZA, H.M.; TORRES, M.A.V.; BACHER, L.B. Árvores exóticas no Brasil: madeireiras, ornamentais e aromáticas. Nova Odessa: Instituto Plantarum de Estudos da Flora, 2003. 384p.

MARIANO, R.S.; PEREIRA, M. Estudo e valoração da arborização de vias públicas do bairro Parque do Trevo na cidade de Ituverava/SP. Nucleus, Ituverava, v.10, n.2, p.105-118, 2013.

MELO, R.R.; FILHO, J.A.L.; JÚNIOR, F.R. Diagnóstico qualitativo e quantitativo da arborização urbana no bairro Bivar Olinto, Patos, Paraíba. Revista da Sociedade Brasileira de Arborização Urbana, Piracicaba, v.2, n.1, p.64-78, 2007.

MILANO, M.S. Avaliação e análise da arborização de ruas de Curitiba. 130f. Dissertação (Mestrado em Engenharia Florestal) - UFPR, Curitiba, 1984. 
MOBOT. Missouri Botanical Garden. Tropicos. Disponível em: <http://www.tropicos.org/> Acesso em: 09 maio 2018.

NEPOMUCENO, D.F.C.; MARINHO, A. B. S.; SILVA, E.T. Identificação e fitossanidade da arborização nas principais praças da cidade de Caratinga, Minas Gerais. Revista de Ciências, Caratinga, v. 7, p. 131-143, 2016.

OLIVEIRA, A.F.; PEREIRA, G.A.; SANTOS, E.; OLIVEIRA, K.D.S.; POMPERMAYER, R.S.; COELHO, S.J.; PEREIRA, J.A.A. Arborização viária conflituosa com a rede elétrica na região oeste de Belo Horizonte - MG. Revista da Sociedade Brasileira de Arborização Urbana, Piracicaba, v.11, n.2, p. 27-44, 2016.

PEREIRA, P.H.; TOPANOTTI, L.R.; DALLACORT, S.; MOTA, C.J.; BRUN, F.G.K.; SILVA, R.T.L. Estudo de caso do risco de queda de árvores urbanas em via pública na cidade de Dois VizinhosPR. Synergismus Scyentifica UTFPR, Pato Branco, v.6, n.1, p.01-10, 2011.

PIRES, N.A.M.T.; MELO, M.S.; OLIVEIRA, D.E.; XAVIER-SANTOS, S. Arborização urbana do município de Goiandira/GO - caracterização quali-quantitativa e proposta de manejo. Revista da Sociedade Brasileira de Arborização Urbana, Piracicaba, v.5, n.3, p.185-205, 2010.

REDIN, C. G.; VOGEL, C.; TROJAHN, C.D.P.; GRACIOLI, C.R.; LONGHI, S.J. Análise da Arborização Urbana em cinco praças do município de Cachoeira do Sul, RS. Revista da Sociedade Brasileira de Arborização Urbana, Piracicaba, v.5, n.3, p. 149-164, 2010.

SAMPAIO, A.C.F.; DUARTE, F.G.; SILVA, E.G.C.; DE ANGELIS, B.L.D.; BLUM, C.T. Avaliação de árvores de risco na arborização de vias públicas de Nova Olímpia, Paraná. Revista da Sociedade Brasileira de Arborização Urbana, Piracicaba, v.5, p.146-167, 2010.

SILVA, A.G.; GONÇALVES, W.; LEITE, H.G.; SANTOS, E. Comparação de três métodos de obtenção de dados para avaliação quali-quantitativa da arborização viária, em Belo HorizonteMG. Revista da Sociedade Brasileira de Arborização Urbana, Piracicaba, v.1, n1, p.31-44, 2006.

SILVA, K.A.R.; LELES. P.S.S.; GIÁCOMO, R.G.; MENDONÇA, B.A.F. Diagnostico e uso de geoprocessamento para manejo da arborização urbana do bairro centro da cidade do Rio de Janeiro - RJ. Revista da Sociedade Brasileira de Arborização Urbana. Piracicaba, v.11, n.4, p.98-114, 2016.

SOUZA, R.C. de; CINTRA, D.P. Arborização viária e conflitos com equipamentos urbanos no bairro da Taquara, RJ. Floresta e Ambiente, Seropédica, v. 14, p. 25-33, 2007.

THE ANGIOSPERM PHYLOGENY GROUP. An update of the Angiosperm Phylogeny Group classification for the orders and families of flowering plants: APG III. Botanical Journal of the Linnean Society, London, n.161, p. 105-121, 2009. 